\title{
AVALIAÇÃO DO PROCESSO DE RESTAURAÇÃO DE ÁREA DE PRESERVAÇÃO PERMANENTE DEGRADADA NO SUL DE MINAS GERAIS
}

\author{
Rafaela Guimarães Silva* \\ Regiane Aparecida Vilas Boas Faria** \\ Lucas Gomes Moreira*** \\ Tiago de Lima Pereira**** \\ Carlos Henrique da Silva***** \\ Soraya Alvarenga Botelho******
}

RESUMO: Estudos que focam a restauração ecológica de Áreas de Preservação Permanente (APP) no entorno de nascentes vêm sendo executados de forma mais intensiva nas últimas décadas devido ao conhecimento da importância desse ecossistema pela sociedade. Protegidas pelo Código Florestal, as zonas ripárias são sistemas com interações complexas que vêm sofrendo grande pressão antropogênica com as ações antrópicas. Diante do exposto, o objetivo geral desse estudo foi avaliar a capacidade de restauração da vegetação ciliar no entorno de uma nascente degradada localizada na sub-bacia do Rio Capivari, município de Lavras (MG). Para a realização da análise fitossociológica foram alocadas 10 parcelas de $10 \mathrm{~m}^{2}$ no interior de uma transecção de $100 \mathrm{~m}$, partindo da origem do olho d'água e seguindo o gradiente de umidade da APP. Com base na análise do estrato regenerante, verificou-se o elevado número de indivíduos de Eremantbus incanus e Vernonanthura phosphorica, espécies da família Asteraceae. A maioria das espécies é pioneira, zoocórica e jovem caracterizando a área como em fase inicial de sucessão mesmo após 09 anos da implementação do plantio de mudas como metodologia de enriquecimento. Tal fato, possivelmente, remete-se a alta biomassa de gramíneas na área estudada, as quais estabelecem vantagem competitiva sobre as espécies nativas. Mesmo pouco significativa, parte da fonte de propágulos da APP provém do banco

Bióloga; Mestre em Tecnologias e Inovações Ambientais na UFLA; Discente de Doutorado do Programa de Pós-Graduação em Ecologia Aplicada da Universidade Federal de Lavras (UFLA), Brasil; E-mail: mgrafaelaguimaraes@yahoo.com.br

** Engenheira Florestal; Doutora em Engenharia Florestal pela UFLA; Docente Substituta no Departamento de Ciências Florestais na Universidade Federal de Lavras (UFLA), Brasil.

*** Engenheiro Agrônomo; Mestre em Tecnologias e Inovações Ambientais na Universidade Federal de Lavras (UFLA), Brasil.

${ }^{* * * *}$ Biólogo; Mestre em Ciências Veterinárias na Universidade Federal de Lavras (UFLA), Brasil.

Mestre em Tecnologias e Inovações Ambientais na Universidade Federal de Lavras (UFLA), Brasil.

${ }^{* * * * * *}$ Engenheira Florestal; Doutora em Engenharia Florestal pela Universidade Federal do Paraná (UFPR); Docente Adjunta Universidade Federal de Lavras (UFLA), Brasil. 
de sementes das mudas plantadas. Portanto, os resultados elucidaram o estado de conservação da APP, mostrando a necessidade de intervenção humana em prol do controle das espécies exóticas invasoras e da melhoria ambiental.

PALAVRAS-CHAVE: Mata Ciliar; Nascente; Regeneração.

\section{EVALUATION OF THE RESTORATION PROCESS OF A DEGRADED PERMANENT PRESERVATION AREA IN THE SOUTH OF MINAS GERAIS, BRAZIL}

ABSTRACT: Studies on the ecological restoration of Permanent Presevation Areas (PPAs) around water sources are being done with regularity during the last years due to importance society is giving to these ecosystems. Riparian zones, protected by the Forest Code, are systems with complex interactivities with great human pressure and anthropic activities. Current paper evaluates the capacity of restoration of riparian vegetation around a degraded water spring in the sub-basin of the river Capivari in the municipality of Larvas MG Brazil. Ten plots measuring $10 \mathrm{~m}^{2}$ within a transection of $100 \mathrm{~m}$, starting from the source and following the PPA's humidity gradient were focused for phytosociological analysis. Foregrounded on the regenerating layer, high numbers of Eremanthus incanus and Vernonanthura phosphorica, species of the family Asteraceae, were reported. Most were pioneer, zoochore and young species which characterized the area as within the initial succession phase even after 9 years of the planting of seedlings for enrichment. The above may have occurred due to the high biomass of grass in the area under analysis which established an advantageous competition on native species. Although slightly relevant, part of the propagules in the PPA derived from seed bank of the planted seedlings. Results showed the conservation state of PPA and the need for human intervention for the control of invading exotic species coupled to environmental improvement.

KEY WORDS: Riparian Vegetation; Water Source; Regeneration.

\section{INTRODUÇÃO}

Os estudos que focam a restauração ecológica de Áreas de Preservação Permanente (APP) no entorno de nascentes vêm sendo executados de forma mais intensiva nas últimas décadas devido ao conhecimento da importância desse 
ecossistema pela sociedade (FARIA et al., 2012; SOUZA et al., 2012; SILVA et al., 2015).

As APPs são as fitofisionomias presentes em um raio de 50 metros em torno de nascentes, as quais são protegidas pelo Código Florestal (Lei Federal $\mathrm{n}^{\mathrm{o}}$ 12.651/2012). As zonas ripárias, em geral, são sistemas com interações hidrológicas, geomorfológicas e biológicas complexas que vêm sofrendo grande pressão antropogênica com a expansão urbana, industrial e atividades agrossilvopastoris (MELO et al., 2013; MARTINS, 2014).

Uma das principais formas de recuperar ambientes perturbados ou degradados é por meio da Restauração Ecológica, que analisa a complexidade dos ecossistemas para a formulação e aprimoramento de técnicas e metodologias (ARONSON et al., 2011). Portanto, para a melhor compreensão acerca delas são necessários mais estudos e aplicações em diversos tipos de áreas sujeitas a diferentes tipos de degradação (CHAZDON, 2008).

Existem diversos métodos de restauração que irão variar com o histórico de uso da terra, estado de degradação do solo, proximidade das fontes de propágulos, presença de agentes dispersores e predação de sementes e plântulas (SCHAEFER, 2009). Além disso, para a elaboração de projetos de restauração ecológica, Lamb et al. (2005) mostraram que os objetivos do proprietário, atividade econômica desenvolvida e o custo da implantação também são critérios a serem considerados no planejamento, uma vez que práticas demoradas e caras muitas vezes não são executadas.

Dentre as metodologias aplicadas em APPs, a condução da regeneração natural (somada à remoção das fontes de degradação) e o plantio de mudas são as mais utilizadas, sendo a primeira a mais utilizada em áreas com capacidade de rebrota e com a presença de remanescentes florestais circunvizinhos que irão fornecer propágulos vegetativos (RODRIGUES et al., 2009). Em contrapartida, o plantio de mudas é usado como técnica de enriquecimento, por exemplo (MARTINS, 2014).

$\mathrm{Na}$ região Sul de Minas Gerais, onde foram aplicadas essas metodologias de restauração, predominam as pequenas propriedades rurais e as atividades agrossilvopastoris, sendo as principais causas de degradação de APPs no entorno de nascentes (FARIA et al., 2012). Apesar das atividades agrossilvopastoris causarem impactos ambientais negativos, dificultando a aplicação das práticas conservacionistas 
(FARIA et al., 2012), o uso de práticas agrícolas sustentáveis em conjunto com as metodologias de Restauração Ecológica podem reduzir os efeitos negativos dessas atividades (CHAZDON, 2008).

Diante do exposto, o objetivo geral desse estudo foi avaliar a capacidade de restauração da vegetação ciliar no entorno de uma nascente degradada por meio da análise estrutural do estrato regenerante, na qual foi implementada a técnica de plantio de mudas há 09 anos.

\section{MATERIAIS E MÉTODOS}

\subsection{CARACTERIZAÇÃO DA ÁREA DE ESTUDO}

O presente estudo foi realizado em uma Área de Preservação Permanente localizada no município de Lavras, Sul do Estado de Minas Gerais, pertencente à subbacia do Rio Capivari, afluente do Rio Grande, nas coordenadas UTM: 23K 510802m E e $7647799 \mathrm{~m}$ N. O relevo da região é suave ondulado, onde predominam os solos Neossolo Flúvico e Argissolo (COSTA, 2004) e um clima do tipo Cwa de Köppen, com temperatura média anual de $19,3{ }^{\circ} \mathrm{C}$ e precipitação média anual de $1530 \mathrm{~mm}$ (SÁ JÚNIOR, 2012).

A nascente foi classificada como pontual - ponto único de escoamento d'água - e degradada, devido à grande biomassa de capim braquiária e presença de trechos de solo exposto (FARIA et al., 2012; SOUZA, 2012). Em dezembro de 2004, a área de estudo correspondente a 0,89 ha foi cercada com arame e mouróes de eucalipto tratado e isolada de distúrbios. Para recuperar a APP foi implementado o método de enriquecimento através do plantio de 225 mudas de 30 espécies nativas (Tabela 1) em um espaçamento de $5 \times 5 \mathrm{~m}$ em linhas de pioneiras e não pioneiras (classificação de grupos ecológicos de acordo com SWAINE \& WHITMORE, 1988).

Todas as análises estatísticas foram realizadas no software Microsoft EXCEL 2007. 
Tabela 1. Relação das Espécies Plantadas na APP e seus Respectivos Grupos Ecológicos (P $=$ Pioneira; $\mathrm{CL}=$ Clímax Exigente de Luz; $\mathrm{CS}=$ Clímax Tolerante à Sombra) e Número de Indivíduos no Plantio

\begin{tabular}{|c|c|c|}
\hline Espécie & Grupo ecológico & $\mathrm{N}^{\mathrm{o}}$ indivíduos no plantio \\
\hline Luebea divaricata & $\mathrm{CL}$ & 6 \\
\hline Machaerium nictitans & CL & 6 \\
\hline Psidium guajava & CL & 6 \\
\hline Aspidosperma parvifolium & CL & 6 \\
\hline Dalbergia nigra & CL & 6 \\
\hline Persea pyrifolia & $\mathrm{CL}$ & 6 \\
\hline Cedrela fissilis & CS & 4 \\
\hline Copaifera langsdorffii & $\mathrm{CL}$ & 4 \\
\hline Syagrus romanzofiana & $\mathrm{CL}$ & 6 \\
\hline Aspidosperma polyneuron & $\mathrm{CL}$ & 6 \\
\hline Myrsine umbellata & CL & 6 \\
\hline Ormosia arborea & CL & 6 \\
\hline Calophyllum brasiliense & CS & 3 \\
\hline Hymenaea coubaril & CS & 4 \\
\hline Cariniana sp. & $\mathrm{CS}$ & 4 \\
\hline Ficus sp. & CS & 4 \\
\hline Myrocarpus frondusus & CS & 4 \\
\hline Inga marginata & CS & 3 \\
\hline Handroanthus serratifolius & CS & 4 \\
\hline Handroanthus impetiginosus & CS & 4 \\
\hline Machaerium villosum & CS & 4 \\
\hline Dendropanax cuneatus & CS & 4 \\
\hline Magnolia ovata & CS & 4 \\
\hline Lithraea molleoides & $\mathrm{P}$ & 16 \\
\hline Schinus terebentifolius & $\mathrm{P}$ & 16 \\
\hline Croton floribundus & $P$ & 16 \\
\hline Solanum granuloso-leprosum & $\mathrm{P}$ & 16 \\
\hline Guazuma ulmifolia & $\mathrm{P}$ & 16 \\
\hline Tapirira guianensis & $\mathrm{P}$ & 16 \\
\hline Croton urucurana & $\mathrm{P}$ & 3 \\
\hline Trema micrantha & $\mathrm{P}$ & 16 \\
\hline Total & & 225 \\
\hline
\end{tabular}


A formação florestal característica da área é Floresta Estacional Semidecidual. A vegetação do entorno da nascente está sob solo hidromórfico, com presença perene de água, criando, assim, um ambiente propício para espécies típicas dessa condição. Adjacente à vegetação nativa presente nesta nascente há um fragmento de mata ciliar que acompanha o fluxo de água e outros pequenos fragmentos florestais em um raio de $500 \mathrm{~m}$. O pequeno fragmento de mata nativa presente no interior da área isolada é delimitado pelo capim braquiária, que na época do plantio, correspondia a $90 \%$ da área estudada.

\subsection{COLETA DE DADOS}

$\mathrm{Na}$ área de estudo, realizou-se o levantamento do estrato regenerante do remanescente florestal alocando 10 parcelas de $1 \times 10 \mathrm{~m}$ no interior de uma transecção de 100 metros, seguindo o gradiente de umidade. Todos os indivíduos com o diâmetro à altura do peito (DAP) $\leq 5 \mathrm{~cm}$ e altura superior a $10 \mathrm{~cm}$ (medido com vara graduada) foram amostrados e coletados para posterior identificação botânica por comparação no Herbário da Universidade Federal de Lavras (Herbário ESAL), por consulta à literatura e a especialistas. A fim de saber a possível fonte de propágulos da regeneração natural, foi realizado o censo dos indivíduos arbóreos ao longo da transecção de 100 metros, cujo critério de inclusão foi medir indivíduos com DAP $\geq 5 \mathrm{~cm}$ e altura superior a $1,30 \mathrm{~m}$.

Os indivíduos do estrato regenerante foram classificados de acordo com os grupos ecológicos propostos por Swaine e Whitmore (1988): pioneiras (P), clímax exigente em luz (CL) e clímax tolerante à sombra (CS). Também foi utilizada outra classificação, quanto à síndrome de dispersão: zoocórica, espécies vegetais que têm suas sementes disseminadas por animais; anemocórica, aquelas cujas sementes são dispersas pelo vento; e autocórica, espécies que dispersam suas sementes pela gravidade ou por deiscência explosiva (PIJL, 1982).

Para avaliar o processo de restauração do estrato regenerante foi feita análise fitossociológica, através de parâmetros específicos que quantificam a presença e a distribuição de cada espécie em relação à área amostrada e em nível de comunidade, tais como: frequência; densidade e dominância, em seus valores 
relativos e absolutos; valor de importância (PEREIRA, 2011); índice de regeneração natural, classes de tamanho da regeneração natural, posição sociológica (FINOL, 1971). Também foram calculados os índices de Shannon (H') e Equabilidade de Pielou para avaliar a diversidade florística do estrato regenerante (BROWER; ZAR, 1984).

\section{RESULTADOS E DISCUSSÃO}

A APP estudada foi classificada como degradada quanto ao seu estado de conservação tendo apenas $23 \%$ da área vegetada e o restante coberto por pastagem. A metodologia de restauração implementada em 2004 (plantio de mudas em 2004) baseou-se no histórico de uso da terra e no nível de degradação da área estudada.

A análise do estrato regenerante fornece informações sobre o processo sucessional da APP acerca da composição florística e do seu atual estado de conservação (SOUZA et al., 2012). A composição florística do estrato regenerante da área estudada, analisada em julho de 2013 (09 anos após o cercamento da área), é apresentada na Tabela 2 .

Tabela 2. Composição Florística do Estrato Regenerante da APP, Localizada no Município de Lavras, Minas Gerais, após 09 anos do Plantio de Mudas e Parâmetros Estruturais: Densidade Absoluta (DA); Densidade Relativa (DR); Frequência Relativa (FR); Classe de Tamanho Relativo da Regeneração Natural (CTRRN); Índice de Regeneração Natural (RN); Grupo Ecológico (GE), onde CL = Clímax Exigente de Luz; CS = Clímax Tolerante à Sombra; e P $=$ Pioneira; e Síndrome de Dispersão (SD), onde Zoo = Zoocórica; Ane = Anemocórica

\begin{tabular}{llcccccccc}
\hline \multicolumn{1}{c}{ Família } & \multicolumn{1}{c}{ Espécie } & $\begin{array}{c}\text { DA } \\
\text { (ind/ha) }\end{array}$ & $\begin{array}{c}\text { DR } \\
(\%)\end{array}$ & $\begin{array}{c}\text { FR } \\
(\%)\end{array}$ & $\begin{array}{c}\text { CTRRN } \\
(\%)\end{array}$ & $\begin{array}{c}\text { RN } \\
(\%)\end{array}$ & VI & GE & SD \\
\hline Malvaceae & Luehea divaricata & 10 & 1,22 & 2,86 & 0,98 & 1,65 & 3,98 & CL & Ane \\
Anacardiaceae & Lithraea molleoides & 40 & 4,88 & 5,71 & 3,78 & 4,72 & 10,37 & P & Zoo \\
Anacardiaceae & Schinus terebentifolius & 10 & 1,22 & 2,86 & 1,73 & 1,91 & 3,98 & P & Zoo \\
Asteraceae & Vernonanthuraphosphorica & 140 & 17,07 & 14,29 & 14,54 & 15,10 & 30,76 & P & Ane \\
Burseraceae & Protium beptaphyllum & 10 & 1,22 & 2,86 & 0,44 & 1,48 & 3,98 & CL & Zoo \\
Asteraceae & Eremanthus incanus & 380 & 46,34 & 20,0 & 53,09 & 39,44 & 65,23 & P & Ane \\
Salicaceae & Casearia sylvestris & 20 & 2,44 & 2,86 & 0,98 & 2,06 & 5,19 & P & Zoo \\
\hline
\end{tabular}


(conclusão)

\begin{tabular}{llllllllll}
\hline Myrtaceae & Psidium guajava & 50 & 6,10 & 8,57 & 7,16 & 7,17 & 14,36 & CL & Zoo \\
Apocynaceae & Aspidosperma parvifolium & 10 & 1,22 & 2,86 & 0,98 & 1,65 & 3,98 & CL & Ane \\
Myrtaceae & Calyptranthes clusiffolia & 20 & 2,44 & 5,71 & 2,71 & 3,56 & 7,97 & CS & Zoo \\
Fabaceae & Machaerium vilosum & 70 & 8,54 & 14,29 & 8,58 & 10,30 & 22,32 & CS & Ane \\
Lecythidaceae & Cariniana legalis & 10 & 1,22 & 2,86 & 0,98 & 1,65 & 3,98 & CL & Ane \\
Anacardiaceae & Mangifera indica & 10 & 1,22 & 2,86 & 0,44 & 1,48 & 3,98 & P & Zoo \\
Malvaceae & Guazuma ulmifolia & 10 & 1,22 & 2,86 & 0,98 & 1,65 & 3,98 & P & Zoo \\
Lauraceae & Nectandra nitidula & 10 & 1,22 & 2,86 & 0,44 & 1,48 & 3,98 & CL & Zoo \\
Myrsinaceae & Myrsine umbellata & 10 & 1,22 & 2,86 & 1,73 & 1,91 & 3,98 & P & Zoo \\
Melastomataceae & Tibouchina sp. & 10 & 1,22 & 2,86 & 0,44 & 1,48 & 3,98 & $\mathrm{P}$ & Ane \\
\hline & Total & 820 & 100 & 100 & 100 & 100 & 100 & & \\
\hline
\end{tabular}

No estrato regenerante foram encontrados 83 indivíduos, 17 espécies, 17 gêneros e 12 famílias.

No censo do estrato arbóreo foram encontradas as espécies Eremanthus incanus, Guazuma ulmifolia, Luehea divaricata, Machaerium vilosum, Lithraea molleoides, Vernonanthura phosphorica, Psidium guajava, Schinus terebentifolius e Tapirira guianensis. As espécies que não são em comum com aquelas que foram utilizadas no plantio foram $V$. phosphorica e E. incanus. Em comparação às 17 espécies listadas na Tabela 1, 09 são comuns àquelas utilizadas no plantio de mudas. Esse resultado demonstra que parte da fonte de propágulos é proveniente do banco de sementes da própria APP fornecido pelo método de enriquecimento.

Baseando-se na composição florística do estrato regenerante da APP e no censo do estrato arbóreo, foi possível observar que a presença de fragmentos florestais nas proximidades contribuiu para a regeneração natural assim como o plantio de mudas, até o presente momento.

As famílias mais representativas foram Anacardiaceae com três espécies e Asteraceae, Malvaceae, Melastomataceae, Myrtaceae com duas espécies cada. A representatividade de espécies de Anacardiaceae também foi encontrada por Ferreira et al. (2011) em nascentes perturbadas/degradadas da bacia do Rio Poxim, em Sergipe. Porém, família mais representativa com relação ao número de indivíduos é Asteraceae que apresentou densidade absoluta de 520 ind.ha $^{-1} \mathrm{e}$ frequência relativa de 34,29\%, cujas espécies foram Eremanthus incanus e Vernonanthura phosphorica. 
De acordo com Rech et al. (2014), essas espécies juntamente com o gênero Bacharis indicam que a APP encontra-se em estágio inicial de regeneração. Essas espécies são adaptadas a áreas abertas onde ocorre maior incidência de luz.

Avaliando o índice de regeneração natural, a espécie $E$. incanus é a mais representativa dentre todas aquelas encontradas no estrato regenerante, representando $46,34 \%$ da comunidade avaliada. Tal observação é comprovada quando também analisados os parâmetros Densidade Relativa (DR) e Valor de Importância (VI) (Tabela 1). Tais parâmetros podem ser justificados pela ocorrência dessa espécie em áreas de transição, entre Floresta Estacional Semidecidual e campos abertos (cerrado), necessitando de luz para germinação de suas sementes e o desenvolvimento de suas plântulas (CRUZ, 2006)

O elevado número de indivíduos de $E$. incanus e $V$. phosphorica na comunidade regenerante evidencia a colonização dessas espécies na APP degradada, principalmente, devido ao fato delas pertencerem ao grupo ecológico das pioneiras. Observou-se que essas espécies são provenientes de outros remanescentes florestais circunvizinhos, uma vez que não foram encontrados indivíduos adultos das mesmas no perímetro da APP. Tais espécies auxiliam na restauração, pois propiciam e criam um ambiente favorável para o estabelecimento de outras espécies de estágios sucessionais mais avançados (FERREIRA et al., 2011).

Essas espécies pioneiras integram o alto valor encontrado para esse grupo ecológico (52,9\%), enquanto das 35,3\% espécies amostradas foram classificadas como clímax exigente em luz e 11,8\% como clímax tolerante à sombra. Souza et al. (2012) encontraram 50\% de espécies climácicas exigentes de luz em nascentes em estágio sucessional médio. A alta porcentagem de espécies pioneiras caracteriza a área como estágio inicial de sucessão juntamente com outras características observadas em campo, tais como: presença de poucos indivíduos arbóreos, alta biomassa de braquiária (Urochloa decumbens) e capim gordura (Melinis minutiflora) entre outros.

Ainda avaliando parâmetros de regeneração natural, a análise da Classe de Tamanho de Regeneração Natural (CTRN) mostrou que a maioria dos indivíduos do estrato regenerante concentra-se na classe de tamanho 2, entre $30 \mathrm{~cm}$ e $1,5 \mathrm{~m}$ de altura. Vale ressaltar que a espécie $E$. incanus se destaca nesta classe, representando 
$66,67 \%$ do total de indivíduos, enquanto que $36,36 \%$ dos indivíduos de $V$. phosphorica apresentam entre 1,5 e 3 metros de altura (classe de tamanho 3).

Os resultados da análise fitossociológica ilustram a importância de $E$. incanus e $V$. phosphorica como espécies propulsoras do processo de restauração não somente pela dominância e densidade, mas também pela colonização do ambiente. Porém, a alta biomassa de braquiária (Urocbloa decumbens) e capim gordura (Melinis minutiflora) está retardando o processo de restauração do ambiente (CORREIA; MARTINS, 2015).

Tabela 3. Indivíduos do Estrato Regenerante Distribuídos em Classes de Tamanho

\begin{tabular}{lc}
\hline Classes de tamanho & \% de indivíduos \\
\hline Classe I - < $30 \mathrm{~cm}$ & 12,05 \\
Classe II - entre 30 e $150 \mathrm{~cm}$ & 46,99 \\
Classe III - entre 150 e $300 \mathrm{~cm}$ & 26,51 \\
Classe IV - > $300 \mathrm{~cm}$ & 14,46 \\
\hline
\end{tabular}

Analisando a frequência relativa e a distribuição das espécies supracitadas na área de estudo, observou-se que a espécie $V$. phosphorica ocorreu na área de maior incidência de luz, antropizada e maior presença de gramíneas; Costa (2004) e Souza et al. (2012), na bacia do Alto Rio Grande, também observaram que V. phosphorica é uma forte competidora e capaz de sobreviver à pressão de gramíneas, estabelecendose em solos pobres e degradados e favorecendo a melhoria ambiental.

A espécie $V$. phosphorica pode contribuir e favorecer o estabelecimento de outras espécies, principalmente arbóreas, mesmo o processo de restauração ocorrendo de forma lenta (SOUZA, 2010). A braquiária e o capim-gordura oferecem poder competitivo sobre as espécies florestais, o que causa grande dificuldade de germinação, sobrevivência e desenvolvimento de espécies nativas advindas da regeneração (SUGANUMA et al., 2013). Isso faz com que essas invasoras agressivas exerçam grande poder e capacidade de impedir que o processo de regeneração natural se estabeleça e desenvolva proporcionando uma diminuição da biodiversidade na vegetação do entorno das nascentes (MARTINS, 2014).

$\mathrm{O}$ índice de diversidade de Shannon $\left(\mathrm{H}^{\prime}\right)$ foi de 1,88 nats.ind $^{-1}$. Esse é um valor considerado abaixo daqueles encontrados por Alvarenga et al. (2006) 
e Souza et al. (2012) em estudos realizados em nascentes degradadas na região do Rio Grande (2,136 e 2,01 nats.ind ${ }^{-1}$, respectivamente). Todos estes valores são considerados baixos quando comparados com outro estudo semelhante realizado por Pinto et al. (2005), cujo índice calculado foi 3,889. Felfili e Resende (2003) observaram que o índice de Shannon varia entre 1,3 e 3,5, alcançando valores até de 4,5 nats.ind ${ }^{-1} \mathrm{em}$ florestas tropicais. Isto é, a diversidade de espécies encontradas no estrato regenerante da APP estudada é baixo quando comparado aos demais estudos com matas ciliares, mesmo o valor encontrado estando no intervalo estimado por Felfili e Resende (2003). A dominância de E. incanus e V. phosphorica também pode estar influenciando nesse valor, uma vez que são espécies abundantes e dominantes no estrato regenerante.

$O$ índice de equabilidade de Pielou da área estudada é 0,665 , próximo ao valor obtido no estudo realizado por Oliveira et al. (2012), que foi de 0,73 . Enquanto isso, Felfili e Resende (2003) encontraram o maior valor de equabilidade de Pielou $(\mathrm{J}$ ') de 0,78 para nascente pontual degradada, o que sugere uniformidade nas proporções do número de indivíduos/número de espécies dentro da comunidade vegetal.

Quanto à síndrome de dispersão, 41,2\% das espécies são anemocóricas e $58,8 \%$ são zoocóricas. A alta porcentagem de espécies zoocóricas reflete a situação encontrada em matas ciliares da Mata Atlântica (PEREIRA, 2006). Nessas áreas, 50\% a 90\% de suas espécies arbóreas são dispersas por animais, logo, observa-se que os animais tornam-se peças fundamentais na restauração de áreas perturbadas interferindo diretamente no tamanho da população vegetal (MARTINS et al., 1995; FRANCO et al., 2014). Os principais animais que contribuem para o sucesso da restauração de ambientes degradados em meio a áreas abertas são as aves e os morcegos que usam árvores vivas e mortas como poleiros e consequentemente contribuindo para a dispersão e formação de banco de sementes (DIAS et al., 2014).

O processo de restauração após 09 anos da implementação do método de enriquecimento não evoluiu significativamente ao ponto de apresentar características de uma área em estágio inicial, como alta biomassa de gramíneas e número elevado de indivíduos de espécies pioneiras e anemocóricas. A presença de espécies exóticas invasoras é devido à atividade econômica predominante na pequena propriedade 
rural que é pecuária, sendo toda a área com uso alternativo do solo formada em pastagem. Cheung et al. (2009) estudaram a relação entre regeneração natural de espécies lenhosas e a presença de gramíneas exóticas em Floresta Atlântica no Sul do Brasil e observaram que a alta biomassa de gramíneas interferiram na riqueza de espécies arbóreas e eram boas competidoras, sendo um entrave para o os processos sucessionais, ocorrendo mais lentamente.

Uma possível solução para o problema exposto seria a intervenção humana com práticas de manutenção da área com capinas, roçadas e coroamento dos indivíduos regenerantes que favorece e auxilia no processo inicial de restauração da vegetação no entorno de nascentes (CHEUNG et al., 2009).

\section{CONSIDERAÇÕES FINAIS}

A avaliação do estrato regenerante e o censo do estrato arbóreo da vegetação ciliar do entorno da nascente permitiu observar o atual estado de conservação da Área de Preservação Permanente que está em fase inicial de sucessão secundária após nove anos da implementação do método de enriquecimento por meio de plantio de mudas. Porém, a incidência de gramíneas invasoras agressivas como capim gordura e braquiária em alta densidade pode estar dificultando o estabelecimento do processo de regeneração natural e a restauração florestal da vegetação ciliar no entorno da nascente.

\section{REFERÊNCIAS}

ALVARENGA, A. P.; BOTELHO, S. A.; PEREIRA, I. M. Avaliação da regeneração natural na recomposição de matas ciliares em nascentes na região sul de Minas Gerais. Revista Cerne, Lavras, v. 12, n. 4, p. 360-372, 2006.

ARONSON, J.; BRANCALION, P. H. S.; DURIGAN, G.; RODRIGUES, R. R.; ENGEL, V. L.; TABARELLI, M.; TOREZAN, J. M. D.; GANDOLFI, S.; MELO, A. C. G.; KAGEYAMA, 
P. Y.; MARQUES, M. C. M.; NAVE, A. G.; MARTINS, S. V.; GANDARA, F. B.; REIS, A.; BARBOSA, L. M.; SCARANO, F. R. What role should government regulation play in ecological restoration?: ongoing debate in São Paulo State, Brazil. Restoration Ecology, v. 19, n. 6, p. 690-695, 2011.

BROWER, J. E.; ZAR, J. H. Field and laboratory methods for general ecology. Dubuque: WM. C. Brow Company Publishers, 1984.

CHAZDON, R. L. Beyond deforestation: restoring forests and ecosystem services on degraded lands. Science, v. 320, n. 5882, p. 1458-1460, 2008.

CHEUNG, K. C.; MARQUES, M. C. M.; LIEBSCH, D. Relação entre a presença de vegetação herbácea e a regeneração natural de espécies lenhosas em pastagens abandonadas na Floresta Ombrófila Densa do Sul do Brasil. Acta botanica brasilica, v. 23, n. 4, p. 1048-1056, 2009.

CORREIA, G. G. S.; MARTINS, S. V. Banco de Sementes do Solo de Floresta Restaurada, Reserva Natural Vale, ES. Floresta e Ambiente, v. 22, n. 1, p. 79-87, 2015.

COSTA, S. S. B. Estudo da bacia do Ribeirão Jaguara - MG, como base para o planejamento da conservação e recuperação das nascentes e matas ciliares. 2004. 214f. Dissertação (Mestrado em Ciências Florestais) - Universidade Federal de Lavras, Lavras, 2004.

CRUZ, E. S. Dinâmica, modelagem do crescimento e cadeia de comercialização da candeia Eremanthus incanus (Less.) Less. 2006. 165f. Tese (Doutorado em Engenharia Florestal) - Universidade Federal de Lavras, Lavras, 2006.

DIAS, C. R.; UMETSU, F.; BREIER, T. B. Contribuição dos poleiros artificiais na dispersão de sementes e sua aplicação na restauração florestal. Ciência Florestal, v. 24, n. 2 , p. 501-507, 2014.

FARIA, R. A. V. B.; BOTELHO, S. A.; SOUZA, L. M. Diagnóstico ambiental de áreas do entorno de 51 nascentes localizadas no município de Lavras, MG. Enciclopédia Biosfera, v. 8, n. 15, p. 648-661, 2012.

FELFILI, J. M.; REZENDE, R. P. Conceitos e métodos em fitossociologia. Brasília: Universidade de Brasília, Departamento de Engenharia Florestal, 2003. 
FERREIRA, R. A.; AGUIAR-NETTO, A. O.; SANTOS, T. I. S.; SANTOS, B. L. E.; MATOS, E. L. Nascentes da sub-bacia hidrográfica do rio Poxim, estado de Sergipe: da degradação à restauração. Revista Árvore, Viçosa, v. 35, n. 2, p. 265-277, 2011.

FINOL, U. H. Nuevos parámetros a considerarse en el analisis estrutural de las selvas virgens tropicales. Revista Forestal Venezolana, Mérida, v. 14, n. 21, p. 337-344, 1971.

FRANCO, B. K. S.; MARTINS, S. V.; FARIA, P. C. L.; RIBEIRO, G. A.; MIRANDA NETO, A. Estrato de regeneração natural de um trecho de Floresta Estacional Semidecidual, Viçosa, MG. Revista Árvore, v. 38, n. 1, p. 31-40, 2014.

LAMB, D.; ERSKINE, P. D.; PARROTA, J. A. Restoration of degraded tropical rain forest landscapes. Science, v. 310, p. 1628-1632, 2005.

MARTINS, S. E.; POMPÉIA, S. L.; ROSSI, L. Síndromes de dispersão de espécies arbóreas da Mata Atlântica de encosta no estado de São Paulo. In: CONGRESSO NACIONAL DE BOTÂNICA, 46., 1995, Ribeirão Preto. Anais... Ribeirão Preto: Sociedade Nacional de Botânica do Brasil, 1995, p. 167.

MARTINS, S. V. Recuperação de matas ciliares: no contexto do Novo Código Florestal. 3. ed. Viçosa: Aprenda Fácil, 2014, v. 1, 220p.

MELO, F. P.; PINTO, S. R.; BRANCALION, P. H.; CASTRO, P. S.; RODRIGUES, R. R.; ARONSON, J.; TABARELLI, M. Priority setting for scaling-up tropical forest restoration projects: Early lessons from the Atlantic Forest Restoration Pact. Environmental Science \& Policy, v. 33, p. 395-404, 2013.

OLIVEIRA, D. G.; FERREIRA, R. A.; MELLO, A. A.; OLIVEIRA, R. S. C. Análise da vegetação em nascentes da bacia hidrográfica do rio Piauitinga, Salgado, SE. Revista Árvore, Viçosa, v. 36, n. 1, p. 127-141, 2012.

PEREIRA, I. M. Estudo da vegetação remanescente como subsídio para a recomposição de áreas ciliares nas cabeceiras do Rio Grande - Minas Gerais. 2006. 278f. Tese (Doutorado em Manejo Ambiental) - Universidade Federal de Lavras, Lavras, 2006. 
PIJL, L. van der. Principles of dispersal in higher plants. 3. ed. New York: Springer Verlag, 1982.

PINTO, L. V. A.; BOTELHO, S. A.; DAVIDE, A. C.; OLIVEIRA-FILHO, A. T. Estudo da vegetação como subsídios para propostas de recuperação das nascentes da bacia hidrográfica do Ribeirão Santa Cruz, Lavras, MG. Revista Árvore, Viçosa, v. 29, n. 5 , p. $775-793,2005$.

RODRIGUES, R. R. R. Pacto pela restauração da mata atlântica: referencial dos conceitos e ações de restauração florestal. Piracicaba: ESALQ/USP, 2009.

SÁ JÚNIOR, A.; CARVALHO, L. G.; SILVA, F. F.; ALVES, M. C. Application of the Köppen classification for climatic zoning in the state of Minas Gerais, Brazil. Theorical and Applied Climatolology, Berlin, v. 108, p. 1-7, apr. 2012.

SCHAEFER, V. Alien invasions, ecological restoration in cities and the loss of ecological memory. Restoration Ecology, Tucson, v. 17, p. 171-176, 2009.

SUGANUMA, M. S.; ASSIS, G. B. D.; MELO, A. C. G. D.; DURIGAN, G. Ecossistemas de referência para restauração de matas ciliares: existem padrões de biodiversidade, estrutura florestal e atributos funcionais? Revista Árvore, v. 37, n. 5, p. 835-847.

SILVA, R. G.; ALVES, M. C.; VILAS BÔAS, R. A.; SARMIENTO, C. M.; BOTELHO, S. A. Monitoramento do processo de Restauração Ecológica de Área de Preservação Permanente por análise espectro-temporal do sensor. In: SIMPOSIO BRASILEIRO DE SENSORIAMENTO REMOTO, 17. (SBSR), 2015, João Pessoa, PB. Anais... São José dos Campos: INPE, 2015, p. 2110-2116. 1 DVD.

SOUZA, L. M. Análise do potencial da regeneração natural do entorno de nascentes em processo de recuperação. 2010. 184f. Dissertação (Mestrado em Engenharia Florestal) - Universidade Federal de Lavras, Lavras, 2010.

SOUZA, L. M.; FARIA, R. A. V. B.; BOTELHO, S. A.; FONTES, M. A. L.; FARIA, J. M. R. Potencial da regeneração natural como método de restauração do entorno de nascente perturbada. Revista Cerne, Lavras, v. 18, n. 4, p. 565-576, 2012. 
SWAINE, M. D.; WHITMORE, T. C. On the definitions of ecological species groups in tropical forest. Vegetation, v. 75, n. 2, p. 81-86, 1988.

Recebido em: 16 de setembro de 2014 Aceito em: 29 de junbo de 2015 